reconstruction (1990-2010s). MR with diffusion-weighted imaging (DWI) (1990-2000s) has been employed most effectively in the detection of recurrent cholesteatoma, particularly in the setting of canal wall up procedures. In some centers, DWI MR has obviated the need for many second look procedures. Dr. Lane will explore the evolution of these radiographic techniques and discuss recent advances in temporal bone imaging, including Photon Counting CT and high field MR (3 T and $7 \mathrm{~T}$ ). These imaging modalities are anticipated to achieve greater degrees of resolution and sensitivity in the detection of acquired and congenital cholesteatomas involving the temporal bone.

Just as imaging techniques have continued to evolve, so have the surgical techniques used to manage cholesteatomas. However, surgical management of cholesteatomas involving the temporal bone continues to be a source of some controversy. Some advocate a one stage procedure with revision of the failures. Others promote modern techniques for post-operative surveillance by employing imaging or endoscopic inspection. Dr. Shelton will discuss the surgical options currently used and review the rationale, controversy and history of the two stage strategy as advocated by the late Jim Sheehy.

Skull base cholesteatomas can be particularly destructive and potentially life threatening. Dr. Arriaga will discuss the traditional and modified versions of skull base neurotologic approaches such as Middle Cranial Fossa, Middle Fossa Transpetrous (extended middle fossa), Retrolabyrinthine, Retrosigmoid and Translabyrinthine approaches. In addition, he will explore the use of four-hand skull base surgery techniques, fallopian bridge strategies and simultaneous application of the endoscope to microscopic visualization to deal with challenging skull base cholesteatomas.

Dr. Poe will bring us "Back to the Future" with an update on the current understanding and treatment of Eustachian tube dysfunction as it relates to chronic otitis media. His focus will be on tubal dilatory dysfunction which typically involves the cartilaginous portion of the Eustachian tube. He will explore the use of Eustachian tuboplasty to treat tubal dysfunction and discuss its potential role in standard tympanoplastic and tympanomastoid procedures.

doi: $10.1017 / \mathrm{S} 0022215116001833$

\section{Back to the future: the evolution of cholesteatoma diagnosis and management (N635)}

\section{ID: 635.2}

\section{Evaluation of Eustachian tube Function and Practical Physiology for Surgeons}

\section{Presenting Author: Dennis Poe}

Dennis Poe

\section{Boston Children's Hospital}

The Eustachian tube (ET) bridges the realm of the nasal cavity and upper aerodigestive tract with the ear and serves to optimize its special sensory role of hearing. The middle ear and mastoid system behaves as an auxiliary sinus and the ET can be thought of as a long, dynamic ostium with a functional valve located within the cartilaginous portion. Failure of the "valve" to function properly can occur if it dilates insufficiently to adequately aerate the middle ear or if it fails to close following dilation. It is affected by all of the same pathophysiologic processes as the nose and other sinuses.

The net effect of the middle ear gas exchange is to cause a constant absorption of gases from the middle ear air space into the venous blood system, creating an ongoing tendency toward developing negative pressure compared to ambient atmospheric pressure. When appropriate, the ET should dilate, typically with a swallow or yawn for about 400 msec, to restore the middle ear pressure toward ambient, optimizing the function of the tympanic membrane. If the dilatory effort is consistently insufficient to adequately aerate the middle ear, ET dilatory dysfunction results with the possible consequences of negative middle ear pressure, retraction of the tympanic membrane, otitis media with effusion, tympanic membrane perforation, conductive hearing loss, fixation of retraction pockets and ultimately cholesteatoma.

Most of the pathology that is responsible for dilatory dysfunction has been observed within the cartilaginous portion and is most commonly due to inflammatory disease, which can be readily diagnosed with transnasal endoscopy. A careful assessment of the dynamics of the ET by endoscopy can be very effective in determining the etiology, location and severity of dilatory and patulous dysfunction within the functional valve in the cartilaginous portion. Vocalizing "K-K-K" demonstrates isolated excursions of the Levator Veli Palatini (LVP) muscle. Swallows start with elevation of the LVP that acts as a scaffold upon which the additional contraction of the Tensor Veli Palatini muscle should be seen to dilate the valve open under normal circumstances. Yawns or vocalizing "Ahhh" can demonstrate a maximal dilatory effort. Disorders of dilation may be observed and classified. Inflammatory disease can be graded on a recently validated mucosal inflammation score instrument. The etiology of the inflammation can be investigated and treated, with the most common causes being infectious or reflux in younger children and over age 6 , allergic disease, reflux, rhinosinusitis, adenoid hypertrophy and other commonly known causes of nasopharyngeal inflammation.

Treatment of the underlying medical conditions can result in improvement of ET function and resolution of middle ear disease. When the medical causes have been optimally treated, but ET dilatory dysfunction persists, possibly due to irreversibly injured mucosa, biofilms or other pathology, tympanostomy tubes are usually recommended. When tubes fail to resolve the problem, treatment of the underlying pathology with surgery can be offered. Surgery may involve turbinate reduction, sinus surgery, adenoidectomy, or balloon dilation of the ET. All of these procedures are designed to remove irreversibly injured tissue and provide a fresh start, assuming the underlying medical conditions are adequately controlled. Failure to control the medical problems can lead to recurrence of inflammatory disease.

Failure of the functional valve to close results in patulous dysfunction. Once thought to be rare, it is now clear that the diagnosis is frequently missed. It can be related to weight loss, chronic illnesses (especially rheumatologic), but it often occurs after long-standing inflammatory dilatory dysfunction with atrophy and decreased mucus production. This may occur particularly with chronic allergic rhinitis. Patients develop frequent sniffing strategies to minimize their symptoms, despite negative middle ear pressure or effusions, raising suspicion that dilatory dysfunction has transitioned to patulous dysfunction. Examination of the tympanic membrane by otoscopy or tympanometry for excursions with ipsilateral nasal breathing can be diagnostic and endoscopy of the ET will reveal a defect in the functional valve, usually within 
the antero-lateral wall. Conservative management is usually successful, but surgical correction is sometimes indicated.

This presentation will show some practical aspects of ET physiology that are relevant to surgeons, methods for evaluating ET function and a systematic approach for diagnosing pathology. Accurate diagnosis of ET disorders will lead to successful management and when appropriate, surgical indications will be clear.

\section{doi:10.1017/S0022215116001845}

\section{Pathogenesis of Cholesteatoma (R636)}

\section{ID: 636.1}

Pathogenesis of the cholesteatoma: changing old concepts

Presenting Author: Leticia Rosito

Leticia Rosito

Hospital de Clínicas de Porto Alegre

Learning Objectives: 1 . To define and classify the cholesteatomas and the spreading routes followed by the disease.; 2. to understand a novel model of pathogenesis with special emphasis on the key role of tympanic membrane retractions; 3. to employ an algorithm to aid the decision making process to maximize surgical results.

Cholesteatoma is a very intriguing condition and still poses a challenge to the otologist. Since it was first described by Duverney in 1683 it has been extensively studied but there are still many pending questions about its development, natural history and prognosis. In 2015 our group proposed a new and embracing classification system for acquired cholesteatomas based on pathogenesis. Our recent studies have shown differences in cholesteatoma growth patterns between children and adults and demonstrated the effect of the disease in the inner ear in both groups. Our contralateral ear studies have also confirmed the essential role of tympanic membrane retractions in the pathogenesis of cholesteatoma.

\section{doi:10.1017/S0022215116001857}

\section{Pathogenesis of Cholesteatoma (R636)}

\section{ID: 636.2}

Congenital Cholesteatoma: Clinical and ethiopatogenetic aspects

Presenting Author: Dragoslava Djeric

Dragoslava Djeric

Medical Faculty University of Belgrade

Learning Objectives: To present the features of congenital cholesteatoma.

\section{Design: Case series}

Patients and Methods: Ten patients were included in the study. The diagnosis of congenital cholesteatoma was based on previous history that excluded tympanic membrane perforation, otorreha, or otologic procedure, an intact tympanic membrane on otomicroscopic examination and a identified cholesteatoma at the time of surgical procedures (tympanotomy, atticotomy, tympanomastoidectomy).

Results: Six of the 10 patients had lesions isolated to the anterosuperior quadrant of the tympanum, the other had more extensive cholesteatoma that involve posterior part of the tympanic cavity and mastoid. Three of the patients underwent surgery for recidivism(none were from isolated anterior lesions). One of these patients was referred at the time of recurrence, one had known residual cholesteatoma, and one had recurrence.

Conclusion: Clinical and surgical findings suggest that congenital cholesteatoma showed various characteristics depending on the location and stage of development.

doi:10.1017/S0022215116001869

\section{Pathogenesis of Cholesteatoma (R636)}

\section{ID: 636.3}

The Pathogenesis of Cholesteatoma Experimental Evidence (R636) 6-6

Presenting Author: Richard Chole

Richard Chole

Washington University in St. Louis School of Medicine

Learning Objectives: The objective of this presentation is to understand the scientific basis for the etiology of aural cholesteatoma.

Over the last century, a number of theories have been proposed to explain the pathogenesis of acquired cholesteatomas. Several of these theories have experimental evidence in animal studies.

Support for the retraction pocket invagination theory is seen in Eustachian tube obstruction models in Mongolian gerbils. When Eustachian tubes of gerbils are ligated in middle ear (bulla) fills with fluid, then over time the pars flaccida retracts, accumulates keratin and forms cholesteatomas.

Support for the epithelial ingrowth theory had been documented in a number of animal models. When toxic materials are applied to the tympanic membrane, destruction of the tympanic membrane and ingrowth of keratinizing epithelium occurs. In infected gerbils cholesteatomas often rupture leading to epithelial ingrowth. Human temporal bone studies have also supported this theory.

The squamous metaplasia theory is not support by experimental evidence. The only demonstration of squamous metaplasia has been seen in vitamin A deficiency. When rats are deprived of dietary vitamin $\mathrm{A}$, the middle ear mucosa changes to a multilayered squamous epithelium, but cholesteatomas have never been seen in this model.

Basal cell hyperplasia and ingrowth through the basal lamina has been observed in human temporal bones for many years. Ruedi first described this phenomenon. It has been observed in human temporal bone section and occurs in spontaneous in induced cholesteatomas and Mongolian gerbils. 